\title{
Deformable models as a tool for biometric and histopathological applications
}

\author{
M.A.G. López*, N.G. Posada*, J.R. Gadelha**, *** and F. Morgado** \\ *INEGI, Faculty of Engineering, University of Porto, Portugal \\ **CESAM \& Department of Biology, University of Aveiro, Portugal \\ ***CAPES Foundation, Ministry of Education of Brazil, Brasília - DF 70040-020, Brazil
}

Mathematical morphology is a novel geometry-based technique for image processing and analysis, originally developed to process binary images, based on the use of simple concepts from set theory and geometry such as set inclusion, intersection, union, complementation, and translation [1,2,3] Geometry is used to represent object shape, physics inflict constraints on how the shape may vary over space and time, and optimal approximation theory make available the formal underpinnings of mechanisms for fitting the models to measured data [1]. Deformable curve, surface, and solid models gained popularity after they were proposed by Terzopoulos for use in computer vision and computer graphics in the mid 1980's introducing the theory of continuous (multidimensional) deformable models in a Lagrangian dynamics setting, based on deformation energies in the form of generalized splines $[1,2,3]$. The deformable model that has attracted the most attention to date is popularly known as "snakes", planar deformable contours that are useful in several image analysis tasks. They are often used to approximate the locations and shapes of object boundaries in images based on the reasonable assumption that boundaries are piecewise continuous or smooth.

This work presents a novel approach since it joins (and, indeed, reinforces) the index framework with the evaluation of the same biological samples by a suitable combination of deformable models. Nucleus contour is identified through Active Shape Models techniques, and cytoplasm contour's detected through parametric Snakes, with prior image preprocessing based on statistical and mathematical morphology techniques. Morphometric parameters such as nucleus and cytoplasm area and ratio between them are then easily computed. Biometry was performed using an ocular micrometer and nucleus/cytoplasm ratios were obtained characterizing each of the three identified stages: Immature, Vitellogenic and Mature. This resulted in a collection of tools, called morphological operators, which are eminently suited for the analysis of shape and structure in binary images. Acartia tonsa was used as model to establish an index for oocyte maturity determination based in citometry and histochemical evaluation of gonadic masses. It was validated the application of a methodology with a realistic background and a new, more accurate and ecologically realistic index for oocyte staging emerged.

\section{References}

1. Tao S., et al., IEEE Transactions on Neural Networks, 18:1424-1432, 2007.

2. Szabo B.K., et al., Academic Radiology, 11:1344-1354, 2004.

3. Kang H.W., Pattern Recognition Letters, 26:2042-2051, 2005. 

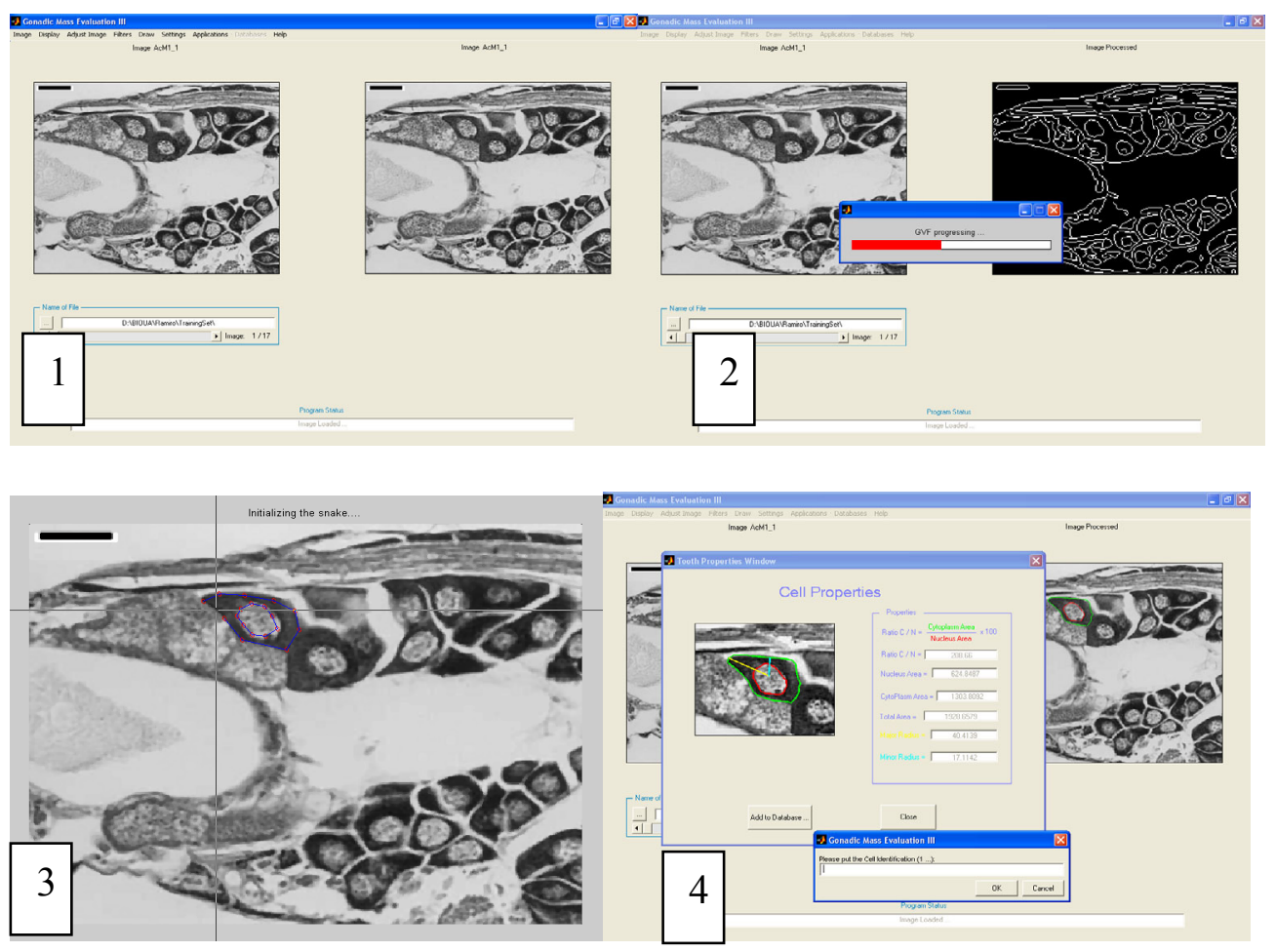

Figure 1. (1) Pre processment; (2) contrast, segmentation and contour; (3) GVF and initial snakes; (4) final snake deformation, micron's measures of each object (area, and diameter).

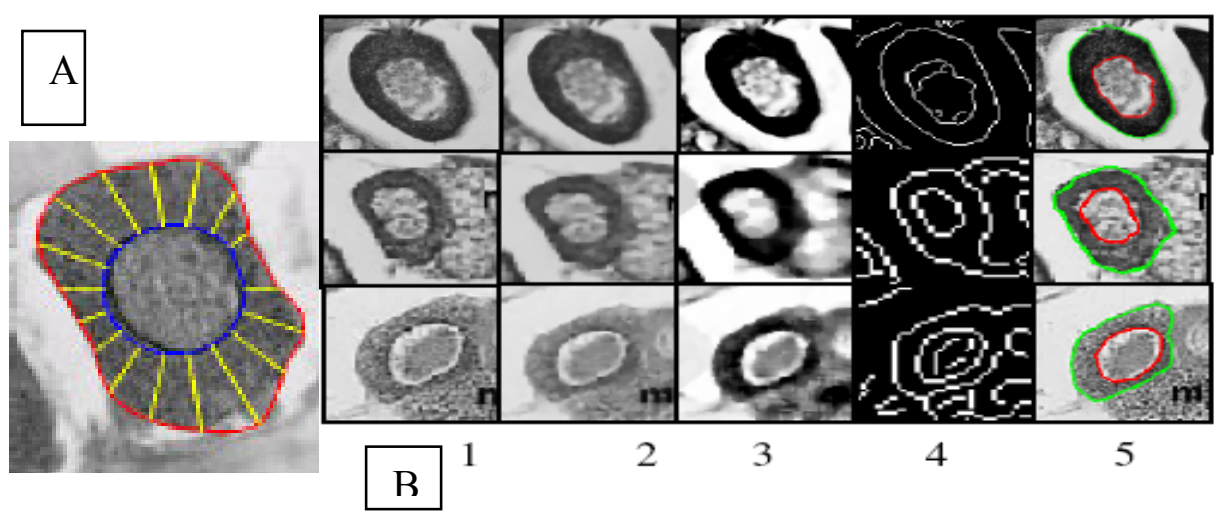

Figure 2 . (A) Spline approximation to create the cytoplasm initial snake; (B) 1-Original image, 2- Enhanced, 3Segmented, 4-Edges, 5-Final cytoplasm and nucleus edges 